\title{
Xenotransplantation of human cultured parathyroid progenitor cells into mouse peritoneum does not induce rejection reaction
}

\author{
IRENEUSZ NAWROT', BOGDAN WOŹNIEWICZ², JACEK SZMIDT ${ }^{1}$, DARIUSZ ŚLADOWSKI', \\ KRZYSZTOF ZAJĄC ${ }^{4}$, WITOLD CHUDZIŃSKI ${ }^{l}$
}

${ }^{1}$ Department of General, Vascular Surgery and Transplantation, Medical University of Warsaw, Warsaw, Poland

${ }^{2}$ Human Parathyroid Biotechnology Center, Warsaw, Poland

${ }^{3}$ Medical University of Warsaw, Warsaw, Poland, and Committee for Advanced Therapies, National Ethics Committee on Animal

Experimentation

${ }^{4}$ Department of General Surgery, Independent Public Healthcare Institution, Piła, Poland

\begin{abstract}
Introduction: Parathyroid progenitor cells devoid of immunogenic antigens were used for human allotransplantation. Although there were many potential reasons for the expiry of transplant activity in humans, we decided to exclude a subclinical form of rejection reaction, and test the rejection reaction in an animal model.

Material and methods: Experiments were carried out on 40 conventional male mice in their third month of life. The animals were housed in groups of 10 per cage in 4 cages with fitted water dispensers and fed a conventional diet based on standard pellet food. They were divided into four groups of 10 animals each, three experimental groups and one control group. Identified progenitor cells were stored in a cell bank. After testing the phenotype, viability, and absence of immunogenic properties, the cells were transplanted into mouse peritoneum cavity.

Results: Animals were observed for 9 weeks. At 9 weeks of observation, the mean serum PTH concentration in the experimental groups was 2.0-2.5 pg/ml, while in the control group it did not exceed $1.5 \mathrm{pg} / \mathrm{ml}$. The immunohistochemical assays demonstrated that millions of viable cells with a phenotype identical to the endocrine cells had survived in the peritoneum. Histologic specimens from different internal organs stained for PTH revealed positive cells labelled with anti-PTH Ab in the intestinal lamina, brain, liver, and spleen.

Conclusions: In the present paper we have demonstrated that xenotransplantation may be used as a model for an explanation of the immunogenic properties of cells generated from postnatal organs for regenerative therapy.
\end{abstract}

Key words: parathyroid progenitor cells, xenotransplantation, rejection.

(Centr Eur J Immunol 2014; 39 (3): 279-284)

\section{Introduction}

In previous studies, we generated and identified progenitor cells from human parathyroids obtained during subtotal resection of the hyperactive glands in dialysed patients with chronic renal insufficiency [1-4]. Progenitor cells devoid of immunogenic antigens were subsequently grafted under the forearm fascia in 293 patients with parathyroid insufficiency (iPTH serum levels below the detection limit). Clinical and laboratory tests performed early after the procedure demonstrated that the transplant was safe, and the serum iPTH and calcium levels normal. Overall, no signs of acute or chronic humoral or cellular rejection were found. However, post-transplantation regeneration time, clinical symptoms and transplant activity differed on a case-by-case basis. We were not able to identify the reason(s) why in some patients the activity of transplanted cells expired after a few months and retransplantation was necessary. The viability of all transplanted cells and the levels of iPTH synthesis were high. There may be many potential reasons for the expiry of transplant activity in humans and we decided to exclude a subclinical form of rejection reaction by performing tests for a rejection reaction in the animal (mouse) model.

Aim of the study was to test the immunogenic properties of human parathyroid progenitor cells in the mouse.

Correspondence: Assoc. Prof. Ireneusz Nawrot, Department of General, Vascular Surgery and Transplantation, Medical University of Warsaw, Banacha 1 A, 02-097 Warsaw, Poland, e-mail: inawrot@post.pl 


\section{Material and methods}

\section{Material}

The experiments were carried out in 40 conventional healthy male mice in their third month of life. The mice were supplied by the licensed animal quarters maintained under veterinary supervision at the Centre for Further Medical Education, Medical University of Warsaw. The animals were housed in groups of 10 per cage in 4 cages with fitted water dispensers and fed a conventional diet based on standard pellet food. They were divided into four groups of 10 animals each, three experimental groups and one control group.

\section{Methods}

\section{Generation and identification of human parathyroid progenitor (stem) cells}

The generation and identification of progenitor (stem) cell population obtained from explants of the parathyroids of donors dialysed due to renal insufficiency were described earlier [1-4]. Samples of blood from the donors were tested for HIV, HBV, HCV and the parathyroid glands underwent histopathological examination. The sterile parathyroids were minced into small 1-mm fragments. The parathyroid explants were generated by in vitro culture in the NUNC $75 \mathrm{~cm}^{3}$ bottles containing $5 \mathrm{~cm}^{3}$ of incubation medium. During the first 2 weeks the parathyroid explants necrotised, with the exception of stromal mesenchymal cells. After 2 weeks, cells with epithelial phenotype, able to synthesize PTH, began to proliferate without additional stimulation. The iPTH concentrations were measured in the supernatant and they proved very high, at over $20 \mathrm{~K} \mathrm{ng} / \mathrm{ml}$, when the bottom of the Falcone bottle $\left(75 \mathrm{~cm}^{2}\right)$ was completely covered by the proliferating cells. That corresponded to about 10 million progenitor cells with the final phenotype characteristic of parathyroid endocrine cells confirmed by cytochemical staining for iPTH. The cells were frozen and banked until 24 hours prior to planned xenotransplantation when they were thawed to reach the temperature of $37^{\circ} \mathrm{C}$ and DMSO was washed out with $1 \mathrm{ml}$ of PBS. The viability of thawed cells was measured with the trypan blue assay. Next the progenitor cells in the amounts of 10 million, 20 million and 30 million were transferred into ampoules. Thus prepared progenitor cells were suspended in $1 \mathrm{ml}$ of physiological saline and administered as a single intraperitoneal injection, as follows: all 10 mice from each of experimental groups 1, 2 and 3 received 10 million, 20 million and 30 million progenitor cells, respectively. Each mouse from the control group was injected with just $1 \mathrm{ml}$ of physiological saline.

\section{Detection of intact PTH}

Intact PTH was assayed using the ELSA-PTH kit from Cisbio Bioassays, Codolet, France. The ELSA PTH is a solid-phase immunoradiometric assay kit. Monoclonal antibodies coated on the solid phase are specific to the mid region and C-terminal part of the PTH. Polyclonal radiolabeled antibodies recognize $\mathrm{N}$-terminal part of the molecule. PTH molecules are sandwiched between the solid phase and tracer. Radioactivity bound to the solid phase is proportional to the concentration of PTH in the sample. Sensitivity of the assay is about $2 \mathrm{pg} / \mathrm{ml}$. Intraand inter-assay coefficient of variation (CV\%) is $5.8 \%$ and $7.9 \%$, respectively. Measurement range is $0.7-1500 \mathrm{pg} / \mathrm{ml}$. Probes with a higher PTH concentration were diluted with the standard $0 \mathrm{pg} / \mathrm{ml}$. The assay measures both PTH 1-84 and PTH 7-84.

\section{Immunocytochemical staining}

Progenitor cell fixation was performed in cold acetone. Cells were exposed to $3 \%$ hydrogen peroxide solution (Sigma) to neutralize endogenous peroxidases. After incubation with a blocking buffer ( $2 \%$ normal serum), the source of secondary Ab, 1\% BSA (Vector), and 2\% Tween 20 were applied for 45-60 minutes at room temperature. Cells were incubated using the Vectastain ABC kit or the Vectastain ABC-AP kit and colour was developed with diaminobenzidine (DAB) substrate solutions. Sections were counterstained with haematoxylin and mounted with Permount (Fisher Scientific).

\section{Results}

Assessment was performed on day 3 post implantation of the progenitor cells and then every 3 weeks until week 9. In the last week of the experiment (week 9), prior to decapitation, iPTH concentrations were measured in the sera. Since the amount of blood from one mouse was very small, in each group iPTH concentrations were measured in the aggregate samples of blood collected from 3 animals. Additionally, at the end of the experiment, various internal organs were taken post mortem from the longest surviving mice for histochemistry to determine whether the cells positive for PTH had migrated from the peritoneum to the perivascular spaces of other organs.

Observation of the behaviour of the animals was the most important in the first 3 days of the experiment since it was expected that rapid or delayed hyperacute rejection may occur. That was why after 3 days one mouse from each of the four groups was sacrificed by decapitation. On opening of the abdominal cavity, no macroscopic changes were detected on the parietal and visceral peritoneum and we speculated that the progenitor parathyroid cells necrotised asymptomatically with no reaction developing in the peritoneal cavity.

At 3, 6 and 9 weeks again no changes were observed in the behaviour of the animals. The procedure for abdominal dissection was modified as it was preceded by an intraperitoneal injection of $5 \mathrm{ml}$ of PBS [5]. After a few minutes 

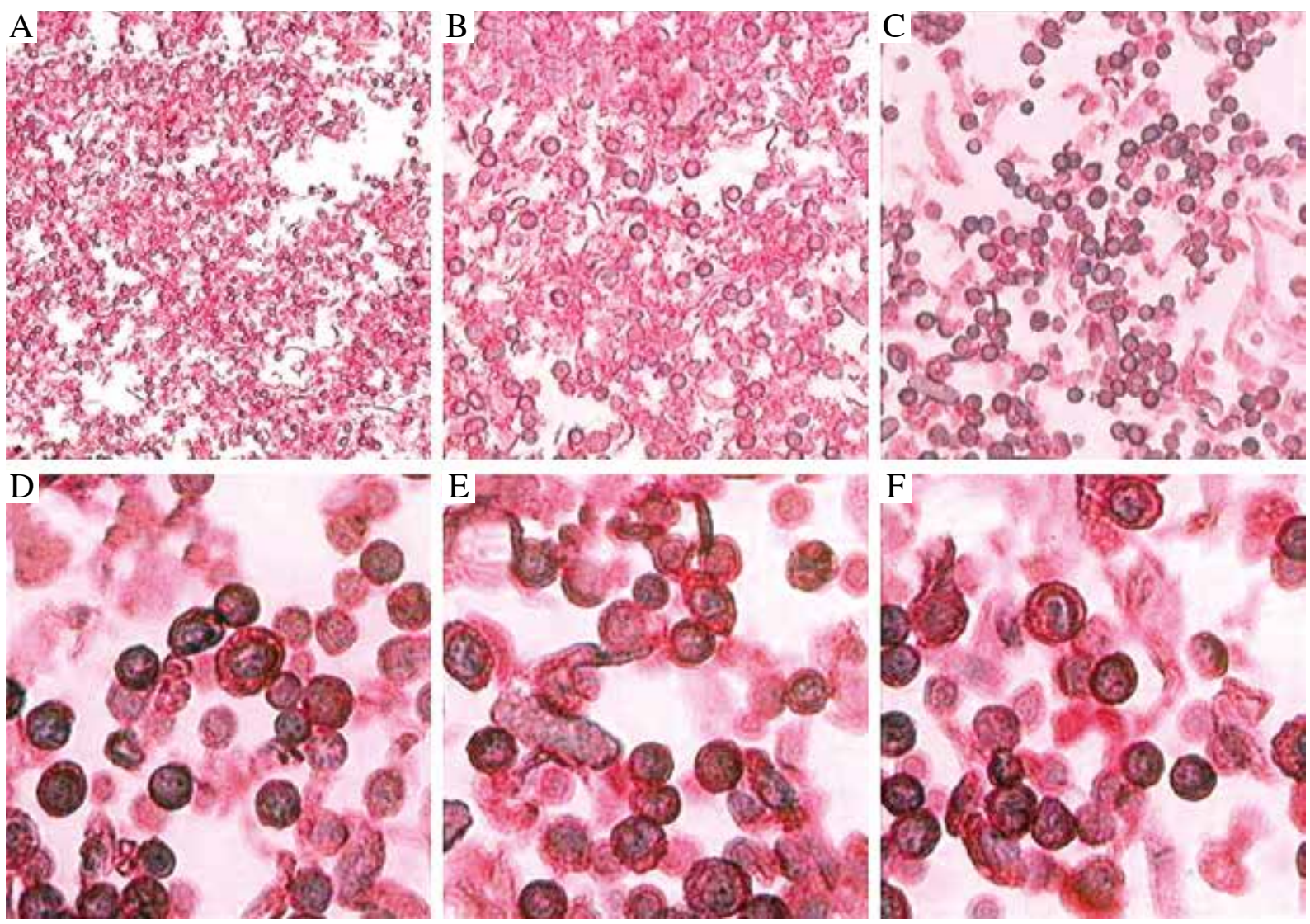

Fig. 1. Immunocytochemical reaction using anti-iPTH antibody. Expression of parathyroid hormone in human PTH cells 9 weeks after xenotransplantation in mouse peritoneum - without rejection. Histochemical staining using avidin-biotin peroxidase complex and anti-PTH monoclonal antibody at $0.5 \mu \mathrm{g} / \mathrm{ml}$ (Abcam). A) magnification 200×, B, C) magnification $200 \times$, D-F) magnification $400 \times$

Table 1. Summary of experimental results

\begin{tabular}{cccccc}
\hline Group & \multicolumn{2}{c}{ Number of progenitor parathyroid cells in the mouse peritoneum } & $\begin{array}{c}\text { Total iPTH (pg/ml) } \\
\text { mouse serum }\end{array}$ \\
\cline { 2 - 6 } & baseline & week 3 & week $\mathbf{6}$ & week 9 & week 9 \\
\hline 1 & $10 \mathrm{M}$ & $12.1 \mathrm{M}$ & $16.5 \mathrm{M}$ & $20.0 \mathrm{M}$ & 2.1 \\
\hline 2 & $20 \mathrm{M}$ & $25.3 \mathrm{M}$ & $34.2 \mathrm{M}$ & $40.0 \mathrm{M}$ & 2.4 \\
\hline 3 & $30 \mathrm{M}$ & $41.3 \mathrm{M}$ & $53.2 \mathrm{M}$ & $60.0 \mathrm{M}$ & 2.5 \\
\hline 4 (control) & 0 & 0 & 0 & 0 & $<1.5$ \\
\hline
\end{tabular}

of peritoneal lavage, the fluid was aspirated and found to be turbid in all experimental groups, but clear in controls. The fluid was preserved for cytologic examination and immunocytochemical assays using anti-PTH, anti-CD4, anti-CD8 and anti-CD 68 antibodies (DAKO Cytomatic). Only positive red staining cytoplasm of PTH-secreting cells was seen in all samples of the effluent from the experimental animals (Fig. 1).

In immunohistochemical assays the progenitor parathyroid cells from all 3 experimental groups were so abun- dant that markers for CD4, CD8, and CD68 cells were undetectable. In the control group, only single CD4-, CD8-, CD68- positive cells were observed.

The assays demonstrated that millions of viable cells with a phenotype identical to the endocrine cells had survived in the peritoneum (Table 1).

At 9 weeks of observation, mice sera were tested for iPTH levels. The mean serum PTH concentration in the experimental groups was $2.0-2.5 \mathrm{pg} / \mathrm{ml}$, while in the control group it did not exceed $1.5 \mathrm{pg} / \mathrm{ml}$ (Table 1). Histologic 
specimens from different internal organs stained for PTH revealed positive cells labelled with anti-PTH Ab in the perivascular spaces of the intestinal lamina, brain, liver, and spleen. At this stage of the study, xenotransplantation confirmed that the activity of progenitor cells generated in our laboratory and grafted in humans should not expire due to rejection reaction. Human cells synthesizing PTH were viable during the observation period and replicated in murine abdominal cavities which served as a natural incubator.

\section{Discussion}

Allogeneic and xenogeneic cells are hypothetical candidates to treat specific diseases and in experimental use. In several fields of medicine, cellular xenotransplantation is slowly emerging as a potential therapeutic tool [6-9]. Xenotransplantation of human cells is applied in many medical disciplines [9-20]. Human glial progenitor cells from aborted foetuses were also transplanted into shiverer mice.

Thymic tissue is plastic, and it is a future challenge to halt or reverse thymic GVHD therapeutically by acting on the level of T-cell progenitors generation, thymic homing, and/or epithelial thymic tissue preservation [21]. For many years the hematopoietic stem cell (HSC) has been well characterized in mice as a cell that can singly reconstitute the whole hematopoietic system of primary recipient animals, as well as that of secondary hosts. Clinical bone marrow transplantation is a successful treatment strategy, and is indirect evidence that such a cell exists in humans $[7,8$, 22-24]. To date, similar criteria have not been applied to human HSCs. Induction of tolerance to xenogeneic antigens has been accomplished only in small animals; however, graft survival in large animal models continues to improve. Further clarification of both the adaptive and innate immune responses to xenogeneic antigens is required for this success to continue [6]. Intracardiac allogeneic porcine mesenchymal stem cells elicit an immune response despite their low immunogenic profile in vitro. This result suggests that the in vivo characteristics of allogeneic MSCs might differ, and emphasizes the importance of pursuing research both in vitro and in vivo $[9,14,15]$. Mesenchymal stem cells (MSCs) are multipotent progenitor cells isolated by various relatively easily accessible tissues, such as bone marrow and umbilical cord blood. Mesenchymal stem cells gained attention because of their ease for in vitro expansion, together with their multilineage potential. More recently, in vitro and in vivo immunosuppressive properties have been ascribed to them as they are able to modulate the function of all major immune cell populations, thus impeding immune responses. The underlying mechanisms of their differentiation and function are not thoroughly understood, but they are still important candidates for tissue regeneration and manipulation of the immune response in graft rejection, graft versus host disease, and autoimmune disorders.

There have been only a few human translational studies performed in the area of stem cell research in patients with chronic obstructive pulmonary disease (COPD) and/ or pulmonary emphysema $[16,17]$. We believe that before progressing to clinical trials with stem cells, human transplantation studies are necessary, otherwise the clinical rationale would be solely based on limited in vitro and animal studies. In the future, stem cell therapy could be a treatment for COPD. Currently, stem cell therapy is still to be considered as an area of active research, lacking a strong rationale for performing clinical trials in COPD. Although stem cells would be likely to represent a heterogeneous population of cells, the different cell subsets and their importance in the pathogenesis of the different clinical phenotypes need to be fully characterised before progressing to clinical trials. Moreover, the potential side effects of stem cell therapy are often underestimated. We should not ignore the fact that some of the most deadly neoplasms arise from stem cells.

Iatrogenic hypoparathyroidism is the most common complication of cervical endocrine surgery. Current management is limited and palliative. As the molecular steps in parathyroid development have been defined, they may be replicable in vitro, with the goal of cellular replacement therapy. Human embryonic stem cell (hESC) lines were investigated as a model for parathyroid regeneration in vitro.

Current knowledge of the immunological and functional aspects of xeno(allo)-cellular transplantation of stem cells, HSC, and umbilical cord blood is used in, e.g. cardiomyopathy, diabetes, liver failure, neural, endocrine and pulmonary diseases, bone regeneration and immunotherapy [9-20, 25-28]. Allotransplantation is promising for parathyroid and thymus reconstruction $[21,29,30]$. On the other hand, transgenic pig cells are probably the best xenogeneic substitute for human application, although basic research on innate and non-innate immunity toward pig cells is still required. In several fields of medicine, cellular xenotransplantation is slowly emerging as a potential therapeutic tool. A number of unexplained doubts as to why the activity of transplanted cells in some patient cells expires without symptoms still exist. In previous papers, a rejection reaction after parathyroid cells transplantation in human was excluded. Recently, we have returned to a hypothesis that a subclinical form of rejection may take place. This was the reason for using an experimental model of human parathyroid cell xenotransplantation.

Replacement of a diseased organ with an autologously derived tissue is an ideal therapy for some medical problems [21, 31]. However, it is difficult to recreate many adult human tissues in vitro due to the functionally necessary architecture of most organs and the lack of understanding of methods to direct the development of the organ of interest. The parathyroid gland is ideal for in vitro organ 
development because this gland is relatively simple, transplantable, and is commonly affected by a surgical complication rather than an autoimmune disease.

Cellular therapies supplement strategies for improving transplant efficiency in the context of cellular therapeutics.

The field of hematopoietic stem cell transplantation (HSCT) has overcome many obstacles, which has led to our current clinical ability to utilize cells collected from bone marrow, mobilized peripheral blood, or umbilical cord blood for the treatment of malignant and non-malignant hematologic diseases. It is in this context that it becomes evident that future progress will lie in our development of the understanding of the biology by which the process of HSCT is regulated. By understanding the cellular components and mechanisms by which HSCT is either enhanced or suppressed it will then be possible to design therapeutic strategies to improve rates of engraftment that will have a positive impact on immune reconstitution postHSCT. In this review we focus primarily on allogeneic hematopoietic stem cell transplantation (allo-HSCT), the current challenges associated with allo-HSCT, and some developing strategies to improve engraftment in this setting $[16,25]$.

\section{Conclusions}

In the present paper we have demonstrated that xenotransplantation may be used as a model for explanation of the immunogenic properties of cells generated from postnatal organs for regenerative therapy.

\section{The authors declare no conflict of interest.}

\section{References}

1. Tolloczko T, Wozniewicz B, Sawicki A, et al. (1994): Cultured parathyroid cell transplantation without immunosuppression in the treatment of surgical hypoparathyroidism. Transplant Proc 26: 1901-1902.

2. Woźniewicz B, Migaj M, Giera B, et al. (1996): Cell culture preparation of human parathyroid cells for allotransplantation without immunosuppression. Transplant Proc 28: 3542-3544.

3. Woźniewicz B, Migaj M, Tołłoczko T, et al. (2001): New trends in cultured parathyroid cells xeno-allo-transplantation. Cell Transplant 10: 528.

4. Nawrot I, Woźniewicz B, Tołłoczko T, et al. (2007): Allotransplantation of cultured parathyroid progenitor cells without immunosuppression: clinical results. Transplantation 83: 734-740.

5. Ray A, Dittel BN (2010): Isolation of mouse peritoneal cavity cells. J Vis Exp 35: 1488.

6. Yamada K, Scalea J (2012): Current progress in xenogeneic tolerance. Curr Opin Organ Transplant 17: 168-173.

7. Ceredig R (2012): When one cell is enough. Stem Cell Res Ther 3: 93-103.
8. Wang H, Yang YG (2012): Innate cellular immunity and xenotransplantation. Curr Opin Organ Transplant 17: 162-167.

9. Poncelet AJ, Denis D, Gianello P (2009): Cellular xenotransplantation. Curr Opin Organ Transplant 14: 168-174.

10. Ablamunits V, Klebanov S, Giese SY, Herold KC (2012): Functional human to mouse adipose tissue xenotransplantation. J Endocrinol 212: 41-47.

11. Burra P, Samuel D, Wendon J, et al. (2004): Strategies for liver support: from stem cell to xenotransplantation. J Hepatol 41: 1050-1059.

12. Wichterle H, Peljto M, Nedelec S (2009): Xenotransplantation of embryonic stem cell-derived motor neurons into develping chick spinal cord. Methods Mol Biol 482: 171-183.

13. Kang EJ, Lee YH, Kim MJ, et al. (2013): Transplantation of porcine umbilical cord matrix mesenchymal stem cells in a mouse model of Parkinson's disease. J Tissue Eng Regen Med 7: 169-182.

14. Poncelet AJ, Hiel AL, Vercruysse J, et al. (2010): Intracardiac allogeneic mesenchymal stem cell transplantation elicits neo-angiogenesis in a fully immunocompetent ischaemic swine model. Eur J Cardiothorac Surg 2010; 38: 781-787.

15. Poncelet AJ, Vercruysse J, Saliez A, Gianello P (2007): Although pig allogeneic mesenchymal stem cells are not immunogenic in vitro, intracardiac injection elicits an immune response in vivo. Transplantation 83: 783-790.

16. Caramori G, Casolari P, Garofano E, et al. (2012): Role of stem cells in the pathogenesis of the chronic obstructive pulmonary disease and the pulmonary emphysema. Recenti Prog Med 103: 31-40.

17. Singhal S, Achary S, Mahajan S, et al. (2011): Stem cells and lung diseases. J Assoc Physicians India 2011; 59: 433-436.

18. Ricardo JR, Gomes JA (2010): Use of stem cells cultured ex vivo for ocular surface reconstruction. Arq Bras Oftalmol 73: 541-547.

19. Parolini O, Soncini M, Evangelista M, Schmidt D (2009): Amniotic membrane and amniotic fluid-derived cells: potential tools for regenerative medicine? Regen Med 4: 275-291.

20. Wei JP, Nawata M, Wakitani S, et al. (2009): Human amniotic mesenchymal cells differentiate into chondrocytes. Cloning Stem Cells 11: 19-26.

21. Shih YR, Kuo TK, Yang AH, et al. (2009): Isolation and characterization of stem cells from the human parathyroid gland. Cell Prolif 42: 461-470.

22. Sotiropoulou PA, Papamichail M (2007): Immune properties of mesenchymal stem cells. Methods Mol Biol 407: 225-243.

23. Popp FC, Eggenhofer E, Renner P, et al. (2009): Mesenchymal stem cells can affect solid organ allograft survival. Transplantation 87 (9 Suppl): S57-62.

24. Le RQ, Melenhorst JJ, Battiwalla M, et al. (2011): Evolution of the donor $\mathrm{T}$-cell repertoire in recipients in the second decade after allogeneic stem cell transplantation. Blood 117: 5250-5256.

25. Jimenez A, Fung HC, Christopherson KW 2nd (2011): Cellular therapies supplement: strategies for improving transplant efficiency in the context of cellular therapeutics. Transfusion 51 Suppl 4: 125S-137S.

26. Bingham EL, Cheng SP, Woods Ignatoski KM, Doherty GM (2009): Differentiation of human embryonic stem cells to a parathyroid-like phenotype. Stem Cells Dev 18: 1071-1080.

27. Abo-Grisha N, Essawy S, Abo-Elmatty DM, Abdel-Hady Z (2013): Effects of intravenous human umbilical cord blood CD34+ stem cell therapy versus levodopa. Arch Med Sci 9: 1138-1151. 
28. Yu B, Ma H, Kong L, et al. (2013): Enhanced connexin 43 expression following neural stem cell transplantation in a rat model of traumatic brain injury. Arch Med Sci 9: 132-138.

29. Wozniewicz B, Janas R, Michalkiewicz J, et al. (2011): Generation and identification of thymic epithelial progenitor cells pTEC by in-vitro processing of human thymic fragments for allotransplantation. Fetal Pediatr Pathol 30: 88-97.

30. Toubert A, Glauzy S, Douay C, Clave E (2012): Thymus and immune reconstitution after allogeneic hematopoietic stem cell transplantation in humans: never say never again. Tissue Antigens 79: 83-89.

31. Woods Ignatoski KM, Bingham EL, Frome LK, Doherty GM. Directed trans-differentiation of thymus cells into parathyroid-like cells without genetic manipulation. Tissue Eng Part C Methods 2011; 17: 1051-1059. 